\title{
Bifidobacterium animalis
}

National Cancer Institute

\section{Source}

National Cancer Institute. Bifidobacterium animalis. NCI Thesaurus. Code C122247.

A species of a Gram-positive, anaerobic, rod-shaped bacterium in the family

Bifidobacteriaceae. B. animalis does not ferment alpha-l-fucose, D-glucuronate,

amylose, arabinogalactan, porcine gastric mucin, arabic gum, ghatti gum, or tragacanth

gum. This bacteria is a normal part of the gut flora and is frequently used as a probiotic. 